\title{
On a Theorem by Florek and Slater on Recurrence Properties of Circle Maps
}

By

\author{
Georg LOHÖFER* and Dieter MAYER**
}

\begin{abstract}
An obviously little known result by Florek and Slater about the exact recurrence times of the sequence $m \beta \bmod 1$ with respect to an arbitrary connected interval $I$ in the unit interval is generalized to disconnected intervals $I_{a, b}=[0, a) \cup(b, 1)$ when $b=1-a, a<1 / 2$. It is shown that the formula of Florek and Slater expressi..g the possible recurrence times in terms of the interval $I$ is valid also in our case. This let us expect that this formula is valid also for general intervals of the form $I_{a, b}$. The relation of this result to the recurrence properties of integrable Hamiltonian systems with two degrees of freedom is obvious.
\end{abstract}

\section{§1. Introduction}

Some time ago when investigating ergodic properties of certain chaotic semiflows with strange attractors [1] we came about a nice but seemingly little known result by Florek [2] and Slater [3] about the distribution of the sequence $\{n \beta \bmod 1\} n \in \mathcal{N}$, for irrational $\beta$ on the unit interval $I=[0,1) . \quad$ Their result concerns the so called gap problem for this sequence: If $I_{a, b}$ denotes the interval $a \leqslant x<b$ in $I$, denote by $N_{\beta}\left(I_{a, b}\right)$ the set $N_{\beta}\left(I_{a, b}\right)=\left\{n \in \mathcal{N}: n \beta \bmod 1 \in I_{a, b}\right\}$. If we arrange the elements of $N_{\beta}\left(I_{a, b}\right)$ according to their order $n_{1}<n_{2}<n_{3}<\cdots$ the gap problem consists in determining the numbers $\tau_{i}=n_{i+1}-n_{i}$ for all $i \in \mathcal{N}$. The rather astonishing result of Florek and Slater then says that for a connected interval $I_{a, b}$ the gaps $\tau_{i}$ can take at most three different values, expressible by the continued fraction expansion of the number $\beta$. This result has a simple interpretation in the theory of dynamical systems: if $R_{\beta}: S_{1} \rightarrow S_{1}$ denotes the map $R_{\beta} \varphi=\varphi+\beta$ of the 1 -sphere $S_{1}=\mathscr{R} / \mathscr{Z}$ into itself we see that the sequence $\{n \beta \bmod 1\}$ is obviously just the orbit of the point $\varphi=0$ under the above map. Hence Florek and Slaters's result describes the recurrence behaviour of the dynamical system $R_{\beta}: S_{1} \rightarrow S_{1}$ with respect to the connected interval $I_{a, b}$ of the 1-sphere. This problem, how the orbit of a system recurs to an arbitrary set of the phase space, arises for arbitrary dynamical systems and

Communicated by M. Kashiwara, June 1, 1988. Revised March 24, 1989.

* Institut für Raumsimulation, DFVIR, D-5000 Köln 90, FRG.

** Heisenberg-Fellow, Institut für Theoretische Physik E, RWTH Aachen, D-5100 Aachen, FRG. 
plays an important role in the foundation of statistical mechanics [4], [5]. In general not much is known about the exact recurrence properties of an arbitrary system, so that it is not too astonishing that even for such a trivial system as the above pure rotation $R_{\beta}$ of $S_{1}$ the problem is not yet completely solved. The first step to such a solution has been done by Florek and Slater who proved

Theorem 0. For any connected interval $I_{a, b}$ in $I$ with $0 \leqslant a<b$ and any irrational $\beta$ there are at most three different gaps $\tau_{1}$ in the set $N_{\beta}\left(I_{a, b}\right)$ :

$$
\begin{aligned}
\tau_{1} & =\min \left\{m: m \beta \bmod 1<b-a=\left|I_{a, b}\right|\right\} \\
\tau_{2} & =\min \left\{m: m \beta \bmod 1>1-\left|I_{a, b}\right|\right\} \\
\tau_{3} & =\tau_{1}+\tau_{2} .
\end{aligned}
$$

The gap $\tau_{3}$ does not arise for all intervals $I_{a, b}$.

In this form the result was announced the first time by Florek in [2] without a proof, which in fact was given for the case of intervals of the form $I_{a, b}$ with $a=0$ by Slater in [3]. He had determined the gaps $\tau_{i}$ in this case already before in [6], expressing them in terms of the best Diophantine approximation denominators $q_{n}$ of the number $\beta$ determined by this number's continued fraction expansion. It is not clear how Flored indeed proved his result (see also a remark by Slater in [3]).

To solve the general gap problem for the rotation $R_{\beta}$ of the 1 -sphere, that means the gaps for any orbil $\left\{R_{\beta}^{n} \varphi\right\}$ for arbitrary $\varphi \in S_{1}$ with respect to an arbitrary connected interval in $S_{1}$, one has obviously to show that the result of Florek and Slater stays true also for intervals of the form $[0, a) \cup(b, 1)$ which are disconnected in $I$ but become connected when regarded as a subset in $S_{1}$. It was not clear to us how Slater's approach in [3] could be applied directly to intervals of the above kind which we denote by $I_{\widehat{a, b}}$. So we had to look for another proof. We found such a proof for the case of intervals of the form $I_{a, 1-a}$ with $a<1 / 2$. In this case we get obviously

$$
R_{\beta}^{n} 0 \in I_{a, 1-a} \text { iff }\|n \beta\|<a,
$$

where the symbol $\|n \beta\|$ denotes the so called Diophantine norm of the irrational $\beta$. The gap problem with respect to the interval $I_{a, 1-a}$ of the sequence $\{n \beta$ $\bmod 1\}$ is then equivalent to the gap problem of the sequence $\{\|n \beta\|\}$ with respect to the interval $I_{0, a}$. This last problem showed up in connection with certain ergodic properties of a class of semiflows studied in [7]: a crucial role there plays the asymptotic behaviour of the function $F_{n}(\lambda)$ for $\operatorname{Re} \lambda>1$ in the limit $n \rightarrow \infty$, where this function is defined as follows

$$
F_{n}(\lambda)=\sum_{m:\left\|q_{n} \beta\right\|<\|m \beta\|<\left\|q_{n-1} \beta\right\|} m^{-\lambda},
$$


where the $q_{n}$ 's are the denominators of the rational approximants of $\beta$ defined by its continued fraction expansion.

If $M_{\varphi}(\beta)$ denotes for $0<\varphi \leqslant 1 / 2$ the set

$$
M_{\varphi}(\beta):=\{m \in \mathcal{N}:|m \beta| \mid<\varphi\}
$$

then we can formulate our main result as

Theorem 1. For any irrational $\beta$ with continued fraction expansion $\beta$ $=\left[b_{0}, b_{1}, b_{2}, \ldots\right]$ and any $\varphi$ with $0<\varphi \leqslant\left\|q_{0} \beta\right\|$ the set $M_{\varphi}(\beta)$ has at most three different gaps. For $a_{i}\left\|q_{i} \beta\right\|+\left\|q_{i+1} \beta\right\|<\varphi \leqslant\left(a_{i}+1\right)\left\|q_{i} \beta\right\|+\left\|q_{i+1} \beta\right\|, 1 \leqslant a_{i}$ $\leqslant b_{i+1}$, there are in the case

a) $2 b_{i+1}>2 a_{i} \geqslant b_{i+1}+1$ exactly the three gaps $q_{i-1}, q_{i}-q_{i-1}, q_{i}$,

b) $2 a_{i} \leqslant b_{i+1}-2$ at most the three gaps $q_{i}, q_{i+1}-2 a_{i} q_{i}, q_{i+1}-\left(2 a_{i}-1\right) q_{i}$ for $\varphi_{i}^{0} \leqslant \varphi \leqslant \varphi_{i}^{1}$, the gaps $q_{i}, q_{i+1}-\left(2 a_{i}+1\right) q_{i}, q_{i+1}-2 a_{i} q_{i}$ for $\varphi_{i}^{1} \leqslant \varphi \leqslant \varphi_{i}^{2}$ and finally the gaps $q_{i}, q_{i+1}-2\left(a_{i}+1\right) q_{i}, q_{i+1}-$ $\left(2 a_{i}+1\right) q_{i}$ for $\varphi_{i}^{2} \leqslant \varphi \leqslant \varphi_{i}^{3}$

c) $2 a_{i}=b_{i+1}$ at most the three gaps $q_{i-1}, q_{i}, q_{i}+q_{i-1} q_{i}$ for $\varphi_{i}^{0} \leqslant \varphi \leqslant \varphi_{i}^{1}$ and the gaps $q_{i-1}, q_{i}-q_{i-1}, q_{i}$ for $\varphi_{i}^{1} \leqslant \varphi \leqslant \varphi_{i}^{3}$

d) $2 a_{i}=b_{i+1}-1$ at most the three gaps $q_{i}, q_{i}+q_{i-1}, q_{i-1}+2 q_{i}$ for $\varphi_{i}^{0} \leqslant \varphi \leqslant \varphi_{i}^{1}$, the gaps $q_{i-1}, q_{i}, q_{i}+q_{i-1}$ for $\varphi_{i}^{1} \leqslant \varphi \leqslant \varphi_{i}^{2}$ and finally the gaps $q_{i}-q_{i-1}, q_{i-1}, q_{i}$ for $\varphi_{i}^{2} \leqslant \varphi \leqslant \varphi_{i}^{3}$

e) $a_{i}=b_{i+1}$ at most the three gaps $q_{i-1}, q_{i}-q_{i-1}, q_{i}$ for $\varphi_{i}^{0} \leqslant \varphi \leqslant \varphi_{i}^{2}$, $q_{i-2}, q_{i-1}-q_{i-2}, q_{i-1}$ for $\varphi_{i}^{2} \leqslant \varphi \leqslant \varphi_{i}^{3}$ if $b_{i}=1$ respectively $q_{i-1}$, $q_{i}-2 q_{i-1}$,

$q_{i}-q_{i-1}$ for $\varphi_{i}^{2} \leqslant \varphi \leqslant \varphi_{i}^{3}$ if $b_{i} \geqslant 2$.

The quantities $\varphi_{i}^{k}$ are thereby defined as follows:

$$
\begin{aligned}
& \varphi_{i}^{0}=a_{i}\left\|q_{i} \beta\right\|+\left\|q_{i+1} \beta\right\|, \\
& \varphi_{i}^{1}=a_{i}\left\|q_{i} \beta\right\|+(l+1)\left\|q_{i+1} \beta\right\|+\left\|q_{i+2} \beta\right\| \text { if } b_{i+2}=2 l+1
\end{aligned}
$$

respectively

$$
\begin{aligned}
& \varphi_{i}^{1}=a_{i}\left\|q_{i} \beta\right\|+l\left\|q_{i+1} \beta\right\|+1 / 2\left(\left\|q_{i+1} \beta\right\|+\left\|q_{i+2} \beta\right\|\right) \text { if } b_{i+2}=2 l \\
& \varphi_{i}^{2}=\left(a_{i}+1\right)\left\|q_{i} \beta\right\|+1 / 2\left\|q_{i+1} \beta\right\| \\
& \varphi_{i}^{3}=\left(a_{i}+1\right)\left\|q_{i} \beta\right\|+\left\|q_{i+1} \beta\right\| .
\end{aligned}
$$

As an immediate consequence of Theorem 1 we get

Corollary 1. There exists a constant $c=c(\lambda)$ such that for any irrational $\beta$, any $\lambda>1$ and any $i \geqslant 1$

$$
F_{i}(\lambda) \leqslant c(\lambda) q_{i}^{-\lambda}
$$

Corollary 2. For any interval $I_{a, 1-a}$ with $a \leqslant\left\|q_{0} \beta\right\|$ the recurrence times $\tau_{i}$ are given by 


$$
\begin{aligned}
\tau_{1} & =\min \left\{m: m \beta \bmod 1<\left|I_{a, 1-a}\right|=2 a\right\} \\
\tau_{2} & =\min \{m: m \beta \bmod 1>1-2 a\} \\
\tau_{3} & =\tau_{1}+\tau_{2}
\end{aligned}
$$

where the last one is, depending on a, not always realized.

This shows that the Florek-Slater formula is also true for this class of disconnected intervals and this let us expect the formula to be valid also for general $I_{\overparen{a, b}}$.

Remark: Our result on the gaps with respect to the interval $I_{\overparen{a, b}-a}$ can be interpreted also as a result on the sequence of gaps arising in the visits of the sequence $m \beta$ mod 1 in the set $[a, 1-a]$. Besides some partial results of Slater on this problem for the interval $[0, a]$ to our knowledge nothing is known for general intervals. The method of our proof relies on the continued fraction expansion of the number $\beta$ and is in its spirit analogous to Slaters approach in $[6]$.

\section{\$2. A Representation of $\|m \beta\|$ in Terms of $\left\|q_{n} \beta\right\|$}

Let us start with some definitions and well known properties of the continued fraction expansion of an irrational number $\beta$ and its relation to the so called Diophantine norms $\|n \beta\|$. If $\beta=\left[b_{0}, b_{1}, \ldots\right]$ is the infinite continued fraction expansion of $\beta$ with $b_{0} \in \mathscr{Z}$ and $b_{i} \in \mathscr{N}$ for $i \geqslant 1$, the $\mathrm{n}$-th principal convergent $p_{n} / q_{n}, n=0,1, \ldots$ is defined by the finite continued fraction $p_{n} / q_{n}$ $=\left[b_{0}, b_{1}, \ldots, b_{n}\right]$. The numbers $p_{n}, q_{n}$ fulfill simple recursion relations [9]:

$$
p_{n+1}=b_{n+1} p_{n}+p_{n-1}
$$

$$
q_{n+1}=b_{n+1} q_{n}+q_{n-1}
$$

with boundary conditions $p_{-1}=1, p_{0}=b_{0}, q_{-1}=0, q_{0}=1$. The Diophantine norms $\|m \beta\|$ of $\beta$ for $m \in \mathscr{N}$ are defined as [10]

$$
\|m \beta\|:=\min _{r \in \mathscr{Z}}|m \beta-r| .
$$

Since $\left|q_{n} \beta-p_{n}\right|=(-1)^{n}\left(\beta_{n+1} q_{n}+q_{n-1}\right)^{-1}$ where $\beta_{n}$ is defined through $\beta=\left[b_{0}\right.$, $\left.b_{1}, \ldots, b_{n-1}, \beta_{n}\right]$ (see [9]) one gets for $n \geqslant 1$ :

$$
\left|q_{n} \beta-p_{n}\right|=\left\|q_{n} \beta\right\| .
$$

For $n=0$ on the other hand one finds

$$
\left|q_{0} \beta-p_{0}\right|= \begin{cases}\left\|q_{0} \beta\right\| & \text { if } b_{i} \geqslant 2 \\ 1-\left\|q_{0} \beta\right\| & \text { if } b_{i}=1\end{cases}
$$


Furthermore the following recursion relations hold:

$$
\begin{gathered}
\left\|q_{n+1} \beta\right\|=-b_{n+1}\left\|q_{n} \beta\right\|+\left\|q_{n-1} \beta\right\| \quad \text { if } n \geqslant 2 \\
\left\|q_{2} \beta\right\|=-b_{2}\left\|q_{1} \beta\right\|+ \begin{cases}\left\|q_{0} \beta\right\| & \text { if } b_{1} \geqslant 2 \\
1-\left\|q_{0} \beta\right\| & \text { if } b_{1}=1,\end{cases}
\end{gathered}
$$

respectively

$$
\left\|q_{1} \beta\right\|= \begin{cases}1-b_{1}\left\|q_{0} \beta\right\| & \text { if } b_{1} \geqslant 2 \\ \left\|q_{0} \beta\right\| & \text { if } b_{1}=1 .\end{cases}
$$

Our first aim is now to express the numbers $\|m \beta\|$ in terms of the best Diophantine approximations $\left\|q_{n} \beta\right\|$, where the $q_{n}$ 's are the best Diophantine approximation denominators determined by the principal convergents (4) of the irrational $\beta$.

To start with we recall the following well known fact [8]:

Lemma 1. If $q_{n}, n=0,1, \ldots$ denote the sequence of denominators of the principal convergents of the irrational number $\beta$ then any integer $m \in \mathcal{N}$ can be uniquely written as

$$
m=\sum_{k=0}^{\infty} r_{k} q_{k}
$$

where the integers $r_{k}$ satisfy the conditions

a) $r_{0} \in\left\{0,1, \ldots, b_{1}-1\right\}$

b) $r_{k} \in\left\{0,1, \ldots, b_{k+1}-1\right\}$ if $r_{k-1} \neq 0$

respectively

$$
r_{k} \in\left\{0,1, \ldots, b_{k+1}\right\} \text { if } r_{k-1}=0 .
$$

Hereby the $b_{i}, i \geqslant 0$, are the partial quotients of $\beta$ :

$$
\beta=\left[b_{0}, b_{1}, b_{2}, \ldots\right] .
$$

We want next derive a similar expansion of the number $\|m \beta\|$ in terms of the $\left\|q_{n} \beta\right\|$. For this let $p_{k}$ be the numerators of the principal convergents $p_{k} / q_{k}$ to $\beta$, defined by its continued fraction expansion. For $m=\sum_{k=0}^{\infty} r_{k} q_{k}$ denote by $\sigma(m)$ the unique integer such that

$$
\left|m \beta-\sum_{k=0}^{\infty} r_{k} p_{k}+\sigma(m)\right|<1 / 2 .
$$

Then obviously 


$$
\|m \beta\|=\left|m \beta-\sum_{k=0}^{\infty} r_{k} p_{k}+\sigma(m)\right|=\left|\sigma(m)+\sum_{k=0}^{\infty}(-1)^{k} r_{k}\left\|q_{k} \beta\right\|\right| .
$$

We can then show

Lemma 2. For all $m \in \mathscr{N}$ with $\|m \beta\| \leqslant\left\|q_{0} \beta\right\|$ the number $\sigma(m)$ vanishes.

Proof. Using the properties of the coefficients $r_{k}$ in expansion (10) of $m$ and the recursion relations (7)-(9)

$$
\begin{aligned}
& \sum_{k=0}^{\infty}(-1)^{k} r_{k}\left\|q_{k} \beta\right\| \leqslant \sum_{j=0}^{\infty} r_{2 j}\left\|q_{2 j} \beta\right\|<\left(b_{1}-1\right)\left\|q_{0} \beta\right\| \\
& +\sum_{j=1}^{\infty} b_{2 j+1}\left\|q_{2 j} \beta\right\|=\left(b_{1}-1\right)\left\|q_{0} \beta\right\|+\left\|q_{1} \beta\right\| \leqslant 1-\left\|q_{0} \beta\right\|,
\end{aligned}
$$

where the strict inequality comes from the fact that for any $m \in \mathscr{N}$ there exists a $k(m)$ such that $r_{k}=0$ for $k>k(m)$. A similar argument as above shows also that

$$
\begin{aligned}
& \sum_{k=0}^{\infty}(-1)^{k} r_{k}\left\|q_{k} \beta\right\| \geqslant-\sum_{j=0}^{\infty} r_{2 j+1}\left\|q_{2 j+1} \beta\right\|>-\sum_{j=0}^{\infty} b_{2 j+2}\left\|q_{2 j+1} \beta\right\| \\
& =-b_{2}\left\|q_{1} \beta\right\|-\left\|q_{2} \beta\right\| \geqslant-\left(1-\left\|q_{0} \beta\right\|\right) .
\end{aligned}
$$

This shows that $\sigma(m) \in\{0,1,-1\}$. Assume that $\sigma(m) \neq 0$ for some $m \in \mathscr{N}$. Then the above estimates imply

$$
\|m \beta\| \geqslant|\sigma(m)|-\left|\sum_{k=0}^{\infty}(-1)^{k} r_{k}\left\|q_{k} \beta\right\|\right| \mid>1-\left(1-\left\|q_{0} \beta\right\|\right)=\left\|q_{0} \beta\right\|
$$

contrary to the assumption $\|m \beta\| \leqslant\left\|q_{0} \beta\right\|$.

The expression $\sum_{k=0}^{\infty}(-1)^{k} r_{k}\left\|q_{k} \beta\right\|$ can be somewhat simplified. If $m$ namely has the expansion

then we fied

$$
m=\sum_{k=k_{0}}^{\infty} r_{k} q_{k}, k_{0}=0, r_{k_{0}} \neq 0
$$

Lemma 3. $\left|\sum_{k=k_{0}}^{\infty}(-1)^{k} r_{k}\left\|q_{k} \beta\right\|\right|=\sum_{k=k_{0}}^{\infty}(-1)^{k-k_{0}} r_{k}\left\|q_{k} \beta\right\|$.

Proof: We only have to show that the right hand side is positive:

$$
\begin{aligned}
& \sum_{k=k_{0}}^{\infty}(-1)^{k-k_{0}} r_{k}\left\|q_{k} \beta\right\| \geqslant r_{k_{0}}\left\|q_{k_{0}} \beta\right\|-\sum_{j=0}^{\infty} r_{k_{0}+1+2 j}\left\|q_{k_{0}+1+2 j} \beta\right\| \\
& >r_{k_{0}}\left\|q_{k_{0}} \beta\right\|-\left(b_{k_{0}+2}-1\right)\left\|q_{k_{0}+1} \beta\right\|-\sum_{j=1}^{\infty} b_{k_{0}+2+2 j}\left\|q_{k_{0}+1+2 j} \beta\right\| \\
& \quad=r_{k_{0}}\left\|q_{k_{0}} \beta\right\|-\left(b_{k_{0}+2}-1\right)\left\|q_{k_{0}+1} \beta\right\|-\left\|q_{k_{0}+2} \beta\right\| .
\end{aligned}
$$


If $k_{0}=0$ then $r_{0} \geqslant 1$ and hence $b_{1} \geqslant 2$. In this case formula (8) gives $\left\|q_{0} \beta\right\|$ $=b_{2}\left\|q_{1} \beta\right\|+\left\|q_{2} \beta\right\|$ and expression (12) is larger than

$$
\left(r_{0}-1\right)\left\|q_{0} \beta\right\|+\left\|q_{1} \beta\right\|>0 .
$$

If on the other hand $k_{0} \geqslant 1$ we get using formula (7)

$$
(12) \geqslant\left(r_{k_{0}}-1\right)\left\|q_{k_{0}} \beta\right\|+\left\|q_{k_{0}+1} \beta\right\|>0 .
$$

This proves Lemma 3.

The results of Lemma 1 to Lemma 3 put together give

Proposition 2. If $m \in \mathscr{N}$ has for irrational $\beta$ the expansion $m=\sum_{k=k_{0}}^{\infty} r_{k} q_{k}$, $r_{k_{0}} \neq 0$ then the Diophantine norms of all $m$ with $\|m \beta\| \leqslant\left\|q_{0} \beta\right\|$ have the representation

$$
\|m \beta\|=\sum_{k=k_{0}}^{\infty}(-1)^{k-k_{0}} r_{k}\left\|q_{k} \beta\right\|
$$

where the $q_{k}$ 's are the denominators of the principal convergents of $\beta$.

Remark: The referee kindly informed us that Prop. 2 is closely related to what in discrepancy problems is called a canonical form as discussed for instance in [11].

\section{§3. Proof of Theorem 1}

The proof will be similar in spirit to the procedure in [6] to solve the gap problem for $\mathrm{m} \beta \bmod 1$. We first discuss certain $\varphi$ 's for for which $M_{\varphi}$ can be determined explicitly and reduce the general problem then to this case. Let us start with the well known fact (see [6], Lemma preceeding Theorem 3) that every number $\varphi$ with $0<\varphi \leqslant\left\|q_{0} \beta\right\|$ can be written uniquely as

$$
\varphi=a_{i}\left\|q_{i} \beta\right\|+\left\|q_{i+1} \beta\right\|+\psi
$$

where the integer $i$ takes values in the set $J_{\beta}$ with $J_{\beta}=\mathcal{N}$ for $b_{1} \geqslant 2$ and $J_{\beta}$ $=\mathscr{N} \backslash\{1\}$ for $b_{1}=1$, the integer $a_{i}$ takes values in $1 \leqslant a_{i} \leqslant b_{i+1}$ and the real number $\psi$ fulfills $0 \leqslant \psi<\left\|q_{i} \beta\right\|$.

The special role played by these numbers was seen already in [6] where the induced partition of the interval $[0,1]$ was used to solve the gap problem for the sequence $m \beta \bmod 1$. If we denote these numbers by $\varphi_{i}=\varphi_{i}\left(a_{i}\right)$ that means

$$
\varphi_{i}\left(a_{i}\right)=a_{i}\left\|q_{i} \beta\right\|+\left\|q_{i+1} \beta\right\|, i \in J_{\beta}
$$

we have 
Proposition 3. The number $m$ belongs to $M_{\varphi_{2}}$ if and only if one of the following canditions holds for the expansion $m=\sum_{k=k_{0}}^{\infty} r_{k} q_{k}, r_{k_{0}} \geqslant 1$ :

$\left(3_{1}\right) \quad k_{0}=i-1: r_{i-1}=1, b_{i+1}-a_{i}<r_{i} \leqslant b_{i+1}-1$

$\left(3_{2}\right) \quad k_{0}=i-1: r_{i-1}=1, r_{i}=b_{i+1}-a_{i}$, there exists $n \geqslant 1$ such that $r_{k}=0$ for $i+1 \leqslant k<i+2 n$ and $r_{i+2 n} \geqslant 1$

$\left(3_{3}\right) \quad k_{0} \geqslant i$ : if $k_{0}=i$ then $0 \leqslant r_{i} \leqslant a_{i}$.

Unfortunately, the partition induced by these $\varphi_{i}$ is not yet fine enough to solve the gap problem for $\|m \beta\|$. So we have to look for finer partitions. For this consider the numbers $\psi_{i}=\psi_{i}\left(a_{i}, c_{i+1}\right)$

$$
\psi_{i}=a_{i}\left\|q_{i} \beta\right\|+c_{i+1}\left\|q_{i+1} \beta\right\|+\left\|q_{i+2} \beta\right\|, i \in J_{\beta}
$$

where the integers $a_{i}$ and $c_{i+1}$ can take the values

$$
1 \leqslant a_{i} \leqslant b_{i+1}-1 \text { respectively } 1 \leqslant c_{i+1} \leqslant b_{i+2},
$$

and therefore are defined only for $b_{i+1} \geqslant 2$. Trivially

$$
\varphi_{i}\left(a_{i}+1\right)>\psi_{i}\left(a_{i}, c_{i+1}\right)>\varphi_{i}\left(a_{i}\right)
$$

In this case we find

Proposition 4. The number $m \in \mathscr{N}$ belongs to $\mathrm{M}_{\psi_{l}}$ if and only if one of the following conditions holds for the expansion $m=\sum_{k=k_{0}} r_{k} q_{k}, r_{k_{0}} \geqslant 1$ :

$\left(4_{1}\right) \quad k_{0}=i-1: r_{i-1}=1, b_{i+1}-a_{i}<r_{i} \leqslant b_{i+1}-1$

$\left(4_{2}\right) \quad k_{0}=i-1: r_{i-1}=1, r_{i}=b_{i+1}-a_{i}, 0 \leqslant r_{i+1} \leqslant c_{i+1}-1$

(4) $k_{0} \geqslant i$ : if $k_{0}=i$ then $0 \leqslant r_{i} \leqslant a_{i}+1$. If $r_{i}=a_{i}+1$ then $b_{i+2}-c_{i+1} \leqslant$ $r_{i+1} \leqslant b_{i+2}-1$. If $r_{i+1}=b_{i+2}-c_{i+1}$ then there exists $n \geqslant 1$ such that $r_{i+k}=0$ for $2 \leqslant k \leqslant 2 n$ and $r_{i+2 n+1} \geqslant 1$.

Because the proof of Prop. 3 is very similar to the proof of Prop. 4 we give only the last one:

Proof of Prop.4. We show first that conditions $\left(4_{1}\right)$ to $\left(4_{3}\right)$ are necessary. If $k_{0} \leqslant i-2$ then by Prop. 2 we get

$$
\begin{aligned}
& \|m \beta\|>\left\|q_{k_{0}} \beta\right\|-\left(b_{k_{0}+2}-1\right)\left\|q_{k_{0}+1} \beta\right\|-\sum_{j=1}^{\infty} b_{k_{0}+2+2 j}\left\|q_{k_{0}+1+2 j} \beta\right\| \\
& =\left\|q_{k_{0}+1} \beta\right\| \geqslant\left\|q_{i-1} \beta\right\|=b_{i+1}\left\|q_{i} \beta\right\|+\left\|q_{i+1} \beta\right\| \geqslant a_{i}\left\|q_{i} \beta\right\|+\left\|q_{i} \beta\right\| \\
& \quad+\left\|q_{i+1} \beta\right\|>a_{i}\left\|q_{i} \beta\right\|+b_{i+2}\left\|q_{i+1} \beta\right\|+\left\|q_{i+2} \beta\right\| \geqslant \psi_{i} .
\end{aligned}
$$

If $k_{0}=i-1$ but $r_{i-1} \geqslant 2$ we find 


$$
\begin{aligned}
& \|m \beta\|>2\left\|q_{i-1} \beta\right\|-\left(b_{i+1}-1\right)\left\|q_{i} \beta\right\|-\sum_{j=1}^{\infty} b_{i+1+2 j}\left\|q_{i 2 j} \beta\right\|=\left\|q_{i-1} \beta\right\| \\
& \quad+\left\|q_{i} \beta\right\|=b_{i+1}\left\|q_{i} \beta\right\|+\left\|q_{i+1} \beta\right\|+\left\|q_{i} \beta\right\|=b_{i+1}\left\|q_{i} \beta\right\| \\
& \quad+\left(b_{i+2}+1\right)\left\|q_{i+1} \beta\right\|+\left\|q_{i+2} \beta\right\|>\psi_{i} .
\end{aligned}
$$

We show next that for $k_{0}=i-1$ and $r_{i-1}=1$ the condition $r_{i} \geqslant b_{i+1}-a_{i}$ is necessary. Assume $r_{i} \leqslant b_{i+1}-a_{i}-1$. Then

$$
\begin{array}{r}
\|m \beta\|>\left\|q_{i-1} \beta\right\|-\left(b_{i+1}-a_{i}-1\left\|q_{i} \beta\right\|-\sum_{j=1}^{\infty} b_{i+1+2 j}\left\|q_{i+2 j} \beta\right\|\right. \\
=\left(a_{i}+1\right)\left\|q_{i} \beta\right\| \geqslant \psi_{i} .
\end{array}
$$

If $k_{0}=i-1$ and $r_{i}=b_{i+1}-a_{i}$ then condition $\left(4_{2}\right)$ is necessary. Otherwise $r_{i+1} \geqslant c_{i+1}$ and we find

$$
\begin{gathered}
\|m \beta\|>\left\|q_{i-1} \beta\right\|-\left(b_{i+1}-a_{i}\right)\left\|q_{i} \beta\right\|+c_{i+1}\left\|q_{i+1} \beta\right\|-\left(b_{i+3}-1\right)\left\|q_{i+2} \beta\right\| \\
-\sum_{j=1}^{\infty} b_{i+3+2 j}\left\|q_{i+2+2 j} \beta\right\|=\psi_{i} .
\end{gathered}
$$

If $k_{0} \geqslant i$ then $r_{i} \leqslant a_{i}+1$ is necessary. This is clear for $a_{i}=b_{i+1}-1$. If $a_{i}$ $<b_{i+1}-1$ assume $r_{i} \geqslant a_{i}+2$. Then

$$
\begin{gathered}
\|m \beta\|>\left(a_{i}+2\right)\left\|q_{i} \beta\right\|-\left(b_{i+2}-1\right)\left\|q_{i+1} \beta\right\|-\sum_{j=1}^{\infty} b_{i+2+2 j}\left\|q_{i+1+2 j} \beta\right\| \\
=\left(a_{i}+1\right)\left\|q_{i} \beta\right\|+\left\|q_{i+1} \beta\right\|>\psi_{i} .
\end{gathered}
$$

If $r_{i}=a_{i}+1$ then $b_{i+2}-c_{i+1} \leqslant r_{i+1} \leqslant b_{i+2}-1$ is necessary. This is clear for $c_{i+1}=b_{i+2}$. For $c_{i+1}<b_{i+2}$ assume $r_{i+1} \leqslant b_{i+2}-c_{i+1}-1$. Then we get

$$
\begin{aligned}
\|m \beta\| & >\left(a_{i}+1\right)\left\|q_{i} \beta\right\|-\left(b_{i+2}-c_{i+1}-1\right)\left\|q_{i+1} \beta\right\|-\sum_{j=1}^{\infty} b_{i+2+2 j}\left\|q_{i+1+2 j} \beta\right\| \\
& =\left(a_{i}+1\right)\left\|q_{i} \beta\right\|-b_{i+2}\left\|q_{i+1} \beta\right\|+\left(c_{i+1}+1\right)\left\|q_{i+1} \beta\right\|-\left\|q_{i+2} \beta\right\| \\
& =a_{i}\left\|q_{i} \beta\right\|+\left(c_{i+1}+1\right)\left\|q_{i+1} \beta\right\|>\psi_{i} .
\end{aligned}
$$

If finally $r_{i}=a_{i}+1$ and $r_{i+1}=b_{i+2}-c_{i+1}$ then there must exist an $n \geqslant 1$ with $r_{i+k}=0$ for $2 \leqslant k \leqslant 2 n$ and $r_{i+2 n+1} \geqslant 1$. Assume on the contrary there exists a $m \geqslant 1$ such that $r_{i+k}=0$ for $2 \leqslant k \leqslant 2 m-1$ but $r_{i+2 m} \geqslant 1$. In this case

$$
\begin{aligned}
& \|m \beta\|>\left(a_{i}+1\right)\left\|q_{i} \beta\right\|-\left(b_{i+2}-c_{i+1}\right)\left\|q_{i+1} \beta\right\|+\left\|q_{i+2 m} \beta\right\| \\
& \quad-\left(b_{i+2 m+2}-1\right)\left\|q_{i+2 m+1} \beta\right\|-\sum_{j=1}^{\infty} b_{i+2 m+2+2 j}\left\|q_{i+2 m+1+2 j} \beta\right\| \\
& =\left(a_{i}+1\right)\left\|q_{i} \beta\right\|+c_{i+1}\left\|q_{i+1} \beta\right\|-b_{i+2}\left\|q_{i+1} \beta\right\|+\left\|q_{i+2 m+1} \beta\right\| \\
& =a_{i}\left\|q_{i} \beta\right\|+c_{i+1}\left\|q_{i+1} \beta\right\|+\left\|q_{i+2} \beta\right\|+\left\|q_{i+2 m+1} \beta\right\|>\psi_{i} .
\end{aligned}
$$


This shows that conditions $\left(4_{1}\right)$ to $\left(4_{3}\right)$ are necessary. Let us next show that they are also sufficient. If the expansion $m=\sum_{k=k_{0}} r_{k} q_{k}$ fulfills condition $\left(4_{1}\right)$ we get

$$
\begin{array}{r}
\|m \beta\|<\left\|q_{i-1} \beta\right\|-\left(b_{i+1}-a_{i}+1\right)\left\|q_{i} \beta\right\|+\left(b_{i+2}-1\right)\left\|q_{i+1} \beta\right\| \\
+\sum_{j=1}^{\infty} b_{i+2+2 j}\left\|q_{i+1+2 j} \beta\right\|=a_{i}\left\|q_{i} \beta\right\|<\psi_{i} .
\end{array}
$$

If $m$ fulfills condition $\left(4_{2}\right)$ we find

$$
\begin{aligned}
& \|m \beta\|<\left\|q_{i-1} \beta\right\|-\left(b_{i+1}-a_{i}\right)\left\|q_{i} \beta\right\|+\left(c_{i+1}-1\right)\left\|q_{i+1} \beta\right\| \\
& \quad+\sum_{j=1}^{\infty} b_{i+2+2 j}\left\|q_{i+1+2 j} \beta\right\|=\left\|q_{i-1} \beta\right\|+a_{i}\left\|q_{i} \beta\right\|-b_{i+1}\left\|q_{i} \beta\right\|+c_{i+1}\left\|q_{i+1} \beta\right\| \\
& \quad-\left\|q_{i+1} \beta\right\|+\left\|q_{i+2} \beta\right\|=a_{i}\left\|q_{i} \beta\right\|+c_{i+1}\left\|q_{i+1} \beta\right\|+\left\|q_{i+2} \beta\right\|=\psi_{i} .
\end{aligned}
$$

If $m$ fulfills condition $\left(4_{3}\right)$ with $k_{0} \geqslant i+1$ we find

$$
\begin{array}{r}
\|m \beta\|<\left\|q_{k_{0}} \beta\right\|+\sum_{j=1}^{\infty} b_{k_{0}+1+2 j}\left\|q_{k_{0}+2 j} \beta\right\|=\left\|q_{k_{0}} \beta\right\|+\left\|q_{k_{0}+1} \beta\right\| \\
\leqslant\left\|q_{i+1} \beta\right\|+\left\|q_{i+2} \beta\right\|<\psi_{i} .
\end{array}
$$

If $m$ fulfills condition $\left(4_{3}\right)$ with $k_{0}=i$ and $r_{i} \leqslant a_{i}$ then

$$
\|m \beta\|<a_{i}\left\|q_{i} \beta\right\|+\left\|q_{i+1} \beta\right\|<\psi_{i} .
$$

If $r_{i}=a_{i}+1$ and $b_{i+2}-c_{i+1}+1 \leqslant r_{r+1} \leqslant b_{i+2}-1$ we get

$$
\begin{aligned}
& \|m \beta\|<\left(a_{i}+1\right)\left\|q_{i} \beta\right\|-\left(b_{i+2}-c_{i+1}+1\right)\left\|q_{i+1} \beta\right\|+\left(b_{i+3}-1\right)\left\|q_{i+2} \beta\right\| \\
& +\sum_{j=1}^{\infty} b_{i+3+2 j}\left\|q_{i+2+2 j} \beta\right\|=a_{i}\left\|q_{i} \beta\right\|+c_{i+1}\left\|q_{i+1} \beta\right\|<\psi_{i} .
\end{aligned}
$$

If finally $r_{i}=a_{i}+1, r_{i+1}=b_{i+2}-c_{i+1}$ and $r_{i+k}=0$ for $2 \leqslant k \leqslant 2 n, r_{i+2 n+1} \geqslant 1$ we find

$$
\begin{gathered}
\|m \beta\|<\left(a_{i}+1\right)\left\|q_{i} \beta\right\|-\left(b_{i+2}-c_{i+1}\right)\left\|q_{i+1} \beta\right\|-r_{i 2 n+1}\left\|q_{i+2 n+1} \beta\right\| \\
+\left(b_{i+2 n+3}-1\right)\left\|q_{i+2 n+2} \beta\right\|+\sum_{j=1}^{\infty} b_{i+2 n+3+2 j}\left\|q_{i+2 n+2+2 j} \beta\right\| \\
\leqslant a_{i}\left\|q_{i} \beta\right\|+c_{i+1}\left\|q_{i+1} \beta\right\|+\left\|q_{i+2} \beta\right\|-\left\|q_{i+2 n+2} \beta\right\|<\psi_{i} .
\end{gathered}
$$

Consider next the numbers $\chi_{i}=\chi_{i}\left(a_{i}\right)$ defined as

$$
\chi_{i}\left(a_{i}\right)=a_{i}\left\|q_{i} \beta\right\|+1 / 2\left\|q_{i+1} \beta\right\|, 1 \leqslant a_{i} \leqslant b_{i+1} .
$$

It is clear that $\chi_{i}\left(a_{i}\right)<\varphi_{i}\left(a_{i}\right)$. 
For the corresponding set $M_{\chi_{2}}$ we find

Proposition 5. $m \in \mathcal{N}$ belongs to $M_{\chi_{2}}\left(a_{i}\right)$ if and only if its expansion $m$ $=\sum_{k=k_{0}} r_{k} q_{k}, r_{k_{0}} \geqslant 1$ fulfills one of the conditions: $\left(5_{1}\right)-\left(5_{3}\right)$ which are conditions $\left(4_{1}\right)-\left(4_{3}\right)$ of Prop. 4 with

$$
\psi_{i}=\psi_{i}\left(a_{i}-1, b_{i+1}\right), \text { or }
$$

$\left(5_{4}\right)$ either $k_{0}=i-1: r_{i-1}=1, \quad r_{i}=b_{i+1}-a_{i}$ and there exists $n \geqslant 1$ with $r_{i+k}=0$ for $1 \leqslant k \leqslant 2 n-1, r_{i+2 n} \geqslant 1$ or

$k_{0}=i: r_{i}=a_{i}$ and there exists $n \geqslant 1$ with $r_{i+k}=0$ for $1 \leqslant k \leqslant 2 n-1$ and $r_{i+2 n} \geqslant 1$.

Remarks: 1$)$ In case $a_{i}=1$ only conditions $\left(5_{3}\right)$ and $\left(5_{4}\right)$ make sense.

2) Condition $\left(5_{4}\right)$ means that exactly one of the two numbers

$$
\begin{aligned}
& m_{1}=q_{i-1}+\left(b_{i+1}-a_{i}\right) q_{i}+\sum_{j=1+2 n} r_{j} q_{j} \\
& m_{2}=a_{i} q_{i}+\sum_{j=1+2 n} r_{j} q_{j}
\end{aligned}
$$

belongs to the set $M_{\chi_{i}}$. This follows from the fact that

$$
\left\|m_{1} \beta\right\|+\left\|m_{2} \beta\right\|=2 a_{i}\|+\| q_{i+1} \beta \| \text { and that }\|m \beta\|=\|n \beta\|
$$

for irrational $\beta$ iff $m=n$.

Finally we need for irrational $\beta$ with $b_{i+2}=2 l_{i+1}$ the special numbers $\zeta_{i}$ $=\zeta_{i}\left(a_{i}, l_{i+1}\right)$ :

$$
\zeta_{i}\left(a_{i}, l_{i+1}\right)=a_{i}\left\|q_{i} \beta\right\|+l_{i+1}\left\|q_{i+1} \beta\right\|+1 / 2\left(\left\|q_{i+1} \beta\right\|+\left\|q_{i+2} \beta\right\|\right) .
$$

They fulfill $\varphi_{i}\left(a_{i}\right)<\zeta_{i}\left(a_{i}, l_{i+1}\right)<\chi_{i}\left(a_{i}+1\right)<\varphi_{i}\left(a_{i}+1\right)$.

For them we find

Proposition 6. The number $m \in \mathscr{N}$ belongs to the set $M_{\zeta_{2}}$ iff one of the following conditions holds for the expansion $m=\sum_{k=k_{0}} r_{k} q_{k}, r_{k_{0}} \geqslant 1$ : Conditions $\left(4_{1}\right)$ to $\left(4_{3}\right)$ of Prop. 4 for $\psi_{i}=\psi_{i}\left(a_{i}, l_{i+1}\right)$, or

$\left(6_{4}\right)$ either $k_{0}=i-1: r_{i-1}, r_{i}=b_{i+1}-a_{i}, r_{i+1}=l_{i+1}$ and there exists $n \geqslant 1$ with $r_{i+k}=0$ for $2 \leqslant k \leqslant 2 n-1$ and $r_{i+2 n} \geqslant 1$

or $k_{0}=i, r_{i}=a_{i}+1, r_{i+1}=l_{i+1}$ and there exists $n \geqslant 1$ such that $r_{i+k}=0$ for $2 \leqslant k \leqslant 2 n-1$ and $r_{i+2 n} \geqslant 1$.

The proofs of all these Propositions run along the lines of the proof of Prop. 4 so that we can omit them here.

Knowing this way the set $M_{\varphi}$ explicitly for certain values of $\varphi$ it is not difficult 
to determine next for these $\varphi$ also the gaps in the sets $M_{\varphi}$.

Proposition 7. For $\beta=\left[b_{0}, b_{1}, \ldots\right]$ irrational and $\varphi=\varphi_{i}\left(a_{i}\right)=a_{i}\left\|q_{i} \beta\right\|$ $+\left\|q_{i+1} \beta\right\|, i \in J_{\beta}$, the set $M_{\varphi}$ has for $2 a_{i} \geqslant b_{i+1}+1$ the gaps $q_{i-1}, q_{i}-q_{i-1}, q_{i}$ and for $2 a_{i} \leqslant b_{i+1}$ the gaps $q_{i}, q_{i+1}-2 a_{i} q_{i}, q_{i+1}-\left(2 a_{i}-1\right) q_{i}$.

Proposition 8. For $\beta$ as in Prop.7 and $\varphi=\chi\left(a_{i}\right)=a_{i}\left\|q_{i} \beta\right\|+1 / 2\left\|q_{i+1} \beta\right\|$ the set $M_{\varphi}$ has for $2 a_{i} \leqslant b_{i+1}$ the gaps $q_{i}, q_{i+1}-\left(2 a_{i}-1\right) q_{i}$. For $b_{i+1}=1$ the set $M_{\varphi}$ has the gaps $q_{i}, q_{i-1}$.

Proposition 9. For $\beta=\left[b_{0}, b_{1}, \ldots\right]$ irrational and $\varphi=\zeta_{i}=a_{i}\left\|q_{i} \beta\right\|$ $+l_{i+1}\left\|q_{i+1} \beta\right\|+1 / 2\left(\left\|q_{i+1} \beta\right\|+\left\|q_{i+2} \beta\right\|\right)$ for $b_{i+2}=2 l_{i+1}$ respectively $\varphi=\bar{\zeta}_{i}$ $=a_{i}\left\|q_{i} \beta\right\|+\left(l_{i+1}+1\right)\left\|q_{i+1} \beta\right\|+\left\|q_{i+2} \beta\right\|$ for $b_{i+2}=2 l_{i+1}+1$ the set $M_{\varphi}$ has for $2 a_{i} \leqslant b_{i+1}$ the gaps $q_{i}, q_{i+1}-2 a_{i} q_{i}$.

The proofs of Prop.7 to 9 are straightforward. Using Prop.3 one determines the right and left nearest neighbours of any point in the set $M$ which determine the gaps. Because this is a rather tedious but very simple task we omit the details hera and refer for more details to the appendix. We are now ready to prove our main result, Theorem 1.

Proof of Theorem 1. Consider the interval $a_{i}\left\|q_{i} \beta\right\|+\left\|q_{i+1} \beta\right\| \leqslant \varphi<\left(a_{i}\right.$ $+1)\left\|q_{i} \beta\right\|+\left\|q_{i+1} \beta\right\|, i \in J_{\beta}, 1 \leqslant a_{i} \leqslant b_{i+1}$. Then any $\varphi$ with $0 \leqslant \varphi \leqslant\left\|q_{0} \beta\right\|$ is contained in one of these intervals.

For $2 b_{i+1}>2 a_{i} \geqslant b_{i+1}+1$ by Prpos.7 there are the three gaps $q_{i-1}, q_{i}$ $-q_{i-1}, q_{i}$. If $a_{i}=b_{i+1}$ the above interval becomes $\left\|q_{i-1} \beta\right\| \leqslant \varphi<\left\|q_{i-1} \beta\right\|$ $+\left\|q_{i} \beta\right\|$. For $\varphi_{i}^{0}=\left\|q_{i-1} \beta\right\|$ the gaps are by Prop. 7 again $q_{i-1}, q_{i}-q_{i-1}$, $q_{i}$. For $\varphi_{i}^{2}=\left\|q_{i-1} \beta\right\|+1 / 2\left\|q_{i} \beta\right\|$ the gaps are by Prop. 8: $q_{i-1}, q_{i}-q_{i-1}$ if $b_{i} \geqslant 2$ respectively $q_{i-1}, q_{i-2}$ for $b_{i}=1$. This shows that for all $\varphi$ with $\varphi_{i}^{0} \leqslant \varphi \leqslant \varphi_{i}^{2}$ the gaps are $q_{i-1}, q_{i}-q_{i-1}, q_{i}$, where the gaps $q_{i}$ disappear one after the other by being divided into two gaps of length $q_{i-1}$ and $q_{i}-q_{i-1}$ at the point $\varphi=\varphi_{i}^{2}$. At the $\varphi$-value $\varphi_{i}^{3}=\left\|q_{i-1} \beta\right\|+\left\|q_{i} \beta\right\|$ by Prop. 7 the gaps are $q_{i-2}, \quad q_{i-1}-q_{i-2}, \quad q_{i-1}$ if $b_{i}=1$ respectively $q_{i-1}, \quad q_{i}-2 q_{i-1}, q_{i}-q_{i-1}$ if $b_{i} \geqslant 2$. This shows that for $\varphi_{i}^{2} \leqslant \varphi \leqslant \varphi_{i}^{3}$ the longest gaps present at $\varphi=\varphi_{i}^{2}$, which are $q_{i}-q_{i-1}$ respectively $q_{i-1}$, are subdivided into two gaps of length $q_{i-1}$ and $q_{i}-2 q_{i-1}$ respectively $q_{i-2}$ and $q_{i-1}-q_{i-2}$. Hence for all $\varphi$ in the interval $\varphi_{i}^{2} \leqslant \varphi \leqslant \varphi_{i}^{3}$ there are only these three resulting gaps present. Therefore part a) and part e) of Theorem 1 are proved.

Let us next discuss part b). For $2 a_{i} \leqslant b_{i+1}-2 \leqslant b_{i+1}$ we see from Prop. 7 that for $\varphi=\varphi_{i}^{0}=a_{i}\left\|q_{i} \beta\right\|+\left\|q_{i+1} \beta\right\|$ there are the gaps $q_{i}, q_{i+1}-2 a_{i} q_{i}, q_{i+1}$ $-\left(2 a_{i}-1\right) q_{i}$. For $\varphi=\varphi_{i}^{1}=a_{i}\left\|q_{i} \beta\right\|+l_{i+1}\left\|q_{i+1} \beta\right\|+1 / 2\left(\left\|q_{i+1} \beta\right\|+\left\|q_{i+2} \beta\right\|\right)$ in case $b_{i+1}=2 l_{i+1}$ respectively $\varphi_{i}^{1}=a_{i}\left\|q_{i} \beta\right\|+\left(l_{i+1}+1\right)\left\|q_{i+1} \beta\right\|+\left\|q_{i+2} \beta\right\|$ for $b_{i+1}=2 l_{i+1}+1$ we see from Prop. 9 that there are the gaps $q_{i}, q_{i+1}$ $-2 a_{i} q_{i}$. This shows that for $\varphi$ in the interval $\varphi_{i}^{0} \leqslant \varphi \leqslant \varphi_{i}^{1}$ all gaps of length 
$q_{i+1}-\left(2 a_{i}-1\right) q_{i}$ disappear step by step by being divided up in gaps of length $q_{i}$ and $q_{i+1}-2 a_{i} q_{i}$. Therefore in this $\varphi$-interval only gaps of length $q_{i}, q_{i+1}$ $-2 a_{i} q_{i}, q_{i+1}-\left(2 a_{i}-1\right) q_{i}$ are present. Because $2\left(a_{i}+1\right) \leqslant b_{i+1}$ we see from Prop. 7 that for $\varphi=\varphi_{i}^{3}=\left(a_{i}+1\right)\left\|q_{i} \beta\right\|+\left\|q_{i+1} \beta\right\|$ there are the gaps $q_{i}, q_{i+1}$ $-2\left(a_{i}+1\right) q_{i}, q_{i+1}-\left(2 a_{i}+1\right) q_{i}$. For $\varphi=\varphi_{i}^{2}=\left(a_{i}+1\right)\left\|q_{i} \beta\right\|+1 / 2\left\|q_{i+1} \beta\right\|$ on the other hand Prop. 8 shows that $M_{\varphi}$ has the gaps $q_{i}, q_{i+1}-\left(2 a_{i}+1\right) q_{i}$. This shows that for $\varphi_{i}^{1} \leqslant \varphi \leqslant \varphi_{i}^{2}$ the gaps of length $q_{i+1}-2 a_{i} q_{i}$ are step by step subdivided into gaps of length $q_{i}$ and $q_{i+1}-\left(2 a_{i}+1\right) q_{i}$ so that in this $\varphi$-interval only the three gaps $q_{i}, q_{i+1}-2 a_{i} q_{i}, q_{i+1}-\left(2 a_{i}+1\right) q_{i}$ are present. In the interval $\varphi_{i}^{2} \leqslant \varphi \leqslant \varphi_{i}^{3}$ finally the gaps of length $q_{i+1}-\left(2 a_{i}+1\right) q_{i}$ are further subdivided into gaps of length $q_{i}$ and $q_{i+1}-\left(2 a_{i}+2\right) q_{i}$ so that for this $\varphi$-interval only the gaps $q_{i}, q_{i+1}-\left(2 a_{i}+1\right) q_{i}, q_{i+1}-\left(2 a_{i}+2\right) q_{i}$ are present. This proves part b) of Theorem 1 .

Let us discuss next part c). If $2 a_{i}=b_{i+1}$ then Prop. 7 shows that for $\varphi_{i}^{0}$ $=a_{i}\left\|q_{i} \beta\right\|+\left\|q_{i+1} \beta\right\|$ there are the gaps $q_{i}, q_{i+1}-2 a_{i} q_{i}, q_{i+1}-\left(2 a_{i}-1\right) q_{i}$. For $\varphi=\varphi_{i}^{1}$ Prop. 9 gives the gaps $q_{i}, q_{i+1}-2 a_{i} q_{i}$ and hence for all $\varphi$ in the interval $\varphi_{i}^{0} \leqslant \varphi \leqslant \varphi_{i}^{1}$ there are the gaps $q_{i}, q_{i+1}-2 a_{i} q_{i}, q_{i+1}-\left(2 a_{i}-1\right) q_{i}$, the last one being step by step divided up into gaps of length $q_{i}, q_{i+1}-2 a_{i} q_{i}$. Because $2 a_{i}$ $=b_{i+1}$ these gaps are therefore $q_{i}, q_{i-1}, q_{i}+q_{i-1}$. For $\varphi=\varphi_{i}^{3}=\left(a_{i}+1\right)\left\|q_{i} \beta\right\|$ $+\left\|q_{i+1} \beta\right\|$ we find $2\left(a_{i}+1\right)=b_{i+1}+1$. Prop. 7 shows then that for this $\varphi$ $=\varphi_{i}^{3}$ we find the gaps $q_{i-1}, q_{i}-q_{i-1}, q_{i}$. For $\varphi_{i}^{1} \leqslant \varphi \leqslant \varphi_{i}^{3}$ the gap $q_{i}$, longest for $\varphi=\varphi_{i}^{1}$, is step by step subdivided into gaps of length $q_{i-1}, q_{i}-q_{i-1}$ which shows that in this interval the gaps are just $q_{i-1}, q_{i}-q_{i-1}$ and $q_{i}$.

Remains to prove part d) of Theorem 1. If $2 a_{i}=b_{i+1}-1$ we find for $\varphi$ $=\varphi_{i}^{0}$ the gaps $q_{i}, q_{i+1}-2 a_{i} q_{i}, q_{i+1}-\left(2 a_{i}-1\right) q_{i}$. For $\varphi=\varphi_{i}^{1}$ Prop. 9 gives the gaps $q_{i}, q_{i+1}-2 a_{i} q_{i}=q_{i-1}+q_{i}$, that means the longest gap at $\varphi=\varphi_{i}^{0}$ disappears by divided up into gaps of length $q_{i}$ and $q_{i+1}-2 a_{i} q_{i}$; hence we find for $\varphi_{i}^{0} \leqslant \varphi \leqslant \varphi_{i}^{1}$ the three gaps $q_{i}, q_{i}+q_{i-1}, 2 q_{i}+q_{i-1}$. Because for $\varphi=\varphi_{i}^{2}$ $=\left(a_{i}+1\right)\left\|q_{i} \beta\right\|+1 / 2\left\|q_{i+1} \beta\right\|$ we have $2 a_{i}=b_{i+1}-1<b_{i+1}$, Prop. 8 gives for this $\varphi$-value the gaps $q_{i-1}, q_{i}$ and hence for $\varphi_{i}^{1} \leqslant \varphi \leqslant \varphi_{i}^{2}$ we have only the gaps $q_{i-1}, q_{i}, q_{i}+q_{i-1}$. For $\varphi=\varphi_{i}^{3}$ finally Prop. 7 gives again the gaps $q_{i-1}, q_{i}$ $-q_{i-1}, q_{i}$ and hence for $\varphi_{i}^{2} \leqslant \varphi \leqslant \varphi_{i}^{3}$ the gaps $q_{i-1}, q_{i}-q_{i-1}, q_{i}$.

This concludes the proof of Theorem 1.

Unfortunately we were not able to treat with our method also those values of $\varphi$ with $\left\|q_{0} \beta\right\| \leqslant \varphi<1 / 2$. We are quite convinced that our results extend also to this case. It would be interesting to extend our results also to rational $\beta$ and to study also the relative frequencies of the different gaps.

Having established the gap structure of the sets $M_{\varphi}$ we are now prepared to prove also Corollary 1. For $i \in J_{\beta}$ the set $M_{i}=\left\{m:\left\|q_{i} \beta\right\|<\|m \beta\|<\left\|q_{i-1} \beta\right\|\right\}$ is obviously contained in the set $M_{\varphi}$ with $\varphi=b_{i+1}\left\|q_{i} \beta\right\|+\left\|q_{i+1} \beta\right\|$ $=\left\|q_{i-1} \beta\right\|$. Since moreover for $\varphi^{\prime}=b_{i+2}\left\|q_{i+1} \beta\right\|+\left\|q_{i+2} \beta\right\|=\left\|q_{i} \beta\right\|$ we have 
$M_{i} \subset \mathscr{C} M_{\varphi^{\prime}} \backslash\left\{q_{i}\right\}$ where

$$
\mathscr{C} M_{\varphi^{\prime}}=\mathscr{N} \backslash M_{\varphi^{\prime}}=\left\{m \in \mathscr{N}:\|m \beta\| \geqslant\left\|q_{i} \beta\right\|\right\} .
$$

Hence $M_{i}=M_{\varphi} \cap\left(\mathscr{C} M_{\varphi^{\prime}} \backslash\left\{q_{i}\right\}\right)$. From these remarks we deduce

Lemma 4. $m \in M_{i}$ if and only if its expansion $m=\sum_{k=k_{0}}^{\infty} r_{k} q_{k}, r_{k_{0}} \geqslant 1$, fulfills one of the following conditions:

(1) $k_{0}=i-1: r_{i-1}=1, b_{i+1}-1 \geqslant r_{i} \geqslant 1$

(2) $k_{0}=i-1: r_{i-1}=1$, there exists $n \geqslant 1$ such that $r_{i+k}=0$ for $0 \leqslant k \leqslant 2 n-1$ but $r_{i+2 n} \geqslant 1$

(3) $k_{0}=i: r_{i}=1$, there exists $n \geqslant 1$ such that $r_{i+k}=0$ for $1 \leqslant k \leqslant 2 n-1$ but $r_{i+2 n} \geqslant 1$.

This Lemma being an immediate consequence of Prop. 3 we can omit the proof. According to Lemma 4 the set $M_{i}$ can be decomposed into three disjoint subsets $M_{i}^{(k)}, k=1,2,3$ defined by the three conditions (1) to (3) of Lemma 4. It is rather trivial to see that every gap within one of these sets is bounded below by $q_{i}$. This implies Corollary 1 as follows:

$F_{i}(\lambda)=\sum_{m \in M_{i}} m^{-\lambda}=\sum_{n=1}^{3} \sum_{m \in M_{i}^{(n)}} m^{-\lambda}$. If $M_{i}^{(n)}=\left\{m_{0}^{(n)}<m_{1}^{(n)}<\cdots\right\}$ we find $m_{0}^{(1)}$ $=q_{i-1}+q_{i}, \quad m_{0}^{(2)}=q_{i-1}+q_{i+2}, \quad m_{0}^{(3)}=q_{i}+q_{i+2} \quad$ and hence $m_{k}^{(n)}=m_{0}^{(n)}$ $+\sum_{j=1}^{k} d_{j, j-1}^{(n)}$, for all $k \geqslant 1$ with $d_{j, j-1}^{(n)}=m_{j}^{(n)}-m_{j-1}^{(n)}$ for all $n=1,2,3$ and all $j \geqslant 1$. Because $d_{j, j-1}^{(n)} \geqslant q_{i}$ and hence $m_{k}^{(k)} \geqslant m_{0}^{(n)}+k q_{i} \geqslant(k+1) q_{i}$ for all $k \geqslant 0$ we get

$$
F_{i}(\lambda) \leqslant \sum_{n=1}^{3} \sum_{k \geqslant 1} k^{-\lambda} q_{i}^{-\lambda}
$$

If therefore $c(\lambda)=3 \sum_{k \geqslant 1} k^{-\lambda}$ we find

$$
F_{i}(\lambda) \leqslant c(\lambda) q_{i}^{-\lambda}
$$

for all $i$ and all irrational $\beta$.

The proof of Corollary 2 follows immediately when comparing our Theorem 1 with the following result of Slater in [6].

Theorem 2(Slater). For $\varphi=a_{i}\left\|q_{i} \beta\right\|+\left\|q_{i+1} \beta\right\|+\psi$, where $0 \leqslant \psi<\left\|q_{i} \beta\right\|$, the gaps of the sequence $\{m \beta \bmod 1\}$ within $\{m \beta \bmod 1<\varphi\}$ are the integers $q_{i}$, $q_{i+1}-a_{i} q_{i}$ and $q_{i+1}-\left(a_{i}-1\right) q_{i}$.

It turns out that the gaps in Theorem 1 are exactly the gaps of the sequence $m \beta$ mod 1 with respect to the cnnected interval $[0,2 a)$. But for such intervals Slater has shown that formulas (1) are valid. But this shows that they are valid 
also for the kind of disconnected intervals $I_{\overparen{a, 1}-\iota}$ we are looking at.

\section{Appendix : Proof of Prop. 7}

For $m \in M_{\Phi_{2}}$ set $m_{L}=\max \left\{m^{\prime} \in M_{\Phi_{2}}: m^{\prime}<m\right\}$ respectively $m_{R}=\min \left\{m^{\prime}\right.$, $\left.m^{\prime} \in M_{\Phi_{i}}: m^{\prime}>m\right\}$. In case the first set is empty we define $m_{L}=0$. The gaps $d_{L}$ and $d_{R}$ are then defined by $d_{L}=m-m_{L}$ and $d_{R}=m_{R}-m$. Theorem 3 shows that the set $M_{\Phi_{1}}$ can be decomposed into disjoint subsets $M_{\Phi_{1}}^{(k)}, k=1,2,3$, where $m \in M_{\Phi_{i}}^{(k)}$ if $m$ satisfies condition $\left(3_{k}\right)$ of Prop.3. For fixed $\Phi_{i}$ define the variable $s_{i}=b_{i+1}-a_{i}$ with $0 \leqslant s_{i+1}-1$. We start our discussion with the case

I. $a_{i}=1, a_{i} \leqslant s_{i}\left(2 a_{i} \leqslant b_{i+1}\right)$

In this case $M_{\Phi_{i}}^{(1)}$ is empty and we have $s_{i} \geqslant 1$ respectively $b_{i+1} \geqslant 2$.

1) Consider $m \in M_{\Phi_{i}}^{(2)}$ given as $m=q_{i-1}+s_{i} q_{i}+\sum_{j=i+2 n} r_{j} q_{j}$.

If $n \geqslant 2$ or $n=1$ and $r_{i+2} \neq b_{i+3}$ we find

$m_{L}=q_{i}+\sum_{j=1+2 n} r_{j} q_{j} \in M_{\Phi_{i}}^{(3)}: d_{L}=q_{i+1}-2 q_{i}=q_{i+1}-2 a_{i} q_{i}$

$m_{R}=q_{i+1}+\sum_{j=1+2 n} r_{j} q_{j} \in M_{\Phi_{i}}^{(3)}: d_{R}=q_{i+1}-\left(2 a_{i}-1\right) q_{i}$.

If $n=1$ and $r_{i+2}=b_{i+3}$ we get

$m_{R}=\left(r_{i+3}+1\right) q_{i+3}+\sum_{j=i+4} r_{j} g_{j} \in M_{\Phi_{i}}^{(3)}: d_{R}=q_{i}$

2) Consider next $m \in M_{\Phi_{1}}^{(3)}, m=\sum_{j=i+n} r_{j} q_{j}, n \geqslant 0$ and $r_{i}=1$ if $n=0$.

If $n=1$ we get

$$
\begin{aligned}
& m_{L}=q_{i}+\left(r_{i+1}-1\right) q_{i+1}+\sum_{j=i+2} r_{j} q_{j} \in M_{\Phi_{i}}^{(3)}: d_{L}=q_{i+1}-\left(2 a_{i}-1\right) q_{i} \\
& m_{R}=q_{i}+\sum_{j=i+1} r_{j} q_{j} \in M_{\Phi_{i}}^{(3)}, \text { if } r_{i+1}<b_{i+2}: d_{R}=q_{i}
\end{aligned}
$$

respectively

$$
\begin{aligned}
m_{R}=\left(r_{i+2 k-1}+1\right) q_{i+2 k-1} & +\sum_{j=i+2 k} r_{j} q_{j} \in M_{\Phi_{i}}^{(3)} \\
\text { where } k & =\min \left\{l: r_{i+2 l-1} \neq b_{i+2 l}\right\}: d_{R}=q_{i} .
\end{aligned}
$$

If $n=0$ we have $m=q_{i}+\sum_{j=i+1} r_{j} q_{j}, l \geqslant 1$, 


$$
\begin{aligned}
& m_{L}=\sum_{j=i+l} r_{j} q_{j} \in M_{\Phi_{i}}^{(3)}: d_{L}=q_{i}, \\
& m_{R}=q_{i-1}+s_{i} q_{i}+\sum_{j=i+l} r_{j} q_{j} \in M_{\Phi_{i}}^{(2)}, \text { if } l \text { is even and } s_{i}=1: \\
& \quad d_{R}=q_{i+1}-2 a_{j} q_{j}
\end{aligned}
$$

respectively

$m_{R}=q_{i+1}+\sum_{j=i+l} r_{j} q_{j}$, if $l$ is odd or $s_{i} \neq 1: d_{R}=q_{i+1}-\left(2 a_{i}-1\right) q_{i}$

II. $a_{i}=1, a_{i} \geqslant s_{i}+1 \quad\left(2 a_{i} \geqslant b_{i+1}+1\right)$

In this case $s_{i}=0$ respectively $b_{i+1}=a_{i}=1$.

1) $m \in M_{\Phi_{i}}^{(2)}, m=q_{i-1}+\sum_{j=2 n} r_{j} q_{j}, n \geqslant 1$ and we find:

$$
\begin{aligned}
& m_{L}=\sum_{j=2 n} r_{j} q_{j} \in M_{\Phi_{i}}^{(3)}: d_{L}=q_{i-1} \\
& m_{R}=q_{i}+\sum_{j=2 n} r_{j} q_{j} \in M_{\Phi_{i}}^{(3)}: d_{R}=q_{i}-q_{i-1}
\end{aligned}
$$

2) $m \in M_{\Phi_{i}}^{(3)}: m=\sum_{j=i+n} r_{j} q_{j}, n \geqslant 0$ and $r_{i}=1$ if $n=0$.

If $n=1$ we find

$$
\begin{aligned}
& m_{L}=q_{i}+\left(r_{i+1}-1\right) q_{i+1}+\sum_{j=i+2} r_{j} q_{j} \in M_{\Phi_{i}}^{(3)}: d_{L}=q_{i+1}-q_{i} \\
& m_{R}=q_{i}+m, \text { if } r_{i+1}<b_{i+2}: d_{R}=q_{i}
\end{aligned}
$$

respectively for $r_{i+1}=b_{i+2}$

$$
m_{R}=\left(r_{i+2}+1\right) q_{i+2}+\sum_{j=i+3} r_{j} q_{j} \in M_{\Phi_{i}}^{(3)}: d_{R}=q_{i}
$$

If $n>1$ and even we find

$$
\begin{aligned}
& m_{L}=\sum_{j=1}^{n / 2} b_{i+2 j} q_{i+2 j-1}+\left(r_{i+n}-1\right) q_{i+n}+\sum_{j=i+n+1} r_{j} q_{j} \in M_{\tilde{\Phi}_{i}}^{(3)}: d_{L}=q_{i} \\
& m_{R}=q_{i-1}+\sum_{j=i+n} r_{j} q_{j} \in M_{\Phi_{1}}^{(2)}: d_{R}=q_{i-1}
\end{aligned}
$$

If $n>1$ and odd

$$
m_{L}=q_{i-1}+\sum_{j=1} b_{i+2 j+1} q_{i+2 j}+\left(r_{i+n}-1\right) q_{i+n}+\sum_{j=i+n+1} r_{j} q_{j} \in M_{\Phi_{i}}^{(2)}: d_{L}=q_{i}
$$




$$
\begin{aligned}
& m_{R}=q_{i}+\sum_{j=i+n} r_{j} q_{j} \in M_{\Phi_{l}}^{(3)}: d_{R}=q_{i} . \\
& \text { If } n=0 \text { and therefore } m=q_{i}+\sum_{j=i+l} r_{j} q_{j}, l \geqslant 1 \text { we get } \\
& m_{L}=q_{i-1}+\sum_{j=i+l} r_{j} q_{j} \in M_{\Phi_{i}}^{(2)} \text { in case } l \text { is even: } d_{L}=q_{i}-q_{i-1} \\
& m_{L}=\sum_{j=i+l} r_{j} q_{j} \in M_{\Phi_{l}}^{(3)} \text { in case } l \text { is odd: } d_{L}=q_{i} \\
& m_{R}=\left(r_{i+1}+1\right) q_{i+1}+\sum_{j=i+2} r_{j} q_{j} \in M_{\Phi_{l}}^{(3)} \text { in case } l=1: d_{R}=q_{i-1}, \\
& m_{R}=q_{i+1}+\sum_{j=i+1} r_{j} q_{j} \in M_{\Phi_{i}}^{(3)} \text { if } l \geqslant 3, \text { or } l=2 \text { and } r_{i+2}<b_{i+3}: d_{R}=q_{i-1} \\
& m_{R}=\left(r_{i+3}+1\right) q_{i+3}+\sum_{j=i+4} r_{j} q_{j} \in M_{\Phi_{i}}^{(3)} \text {, if } l=2 \text { and } r_{i+2}=b_{i+3}: d_{R}=q_{i-1}
\end{aligned}
$$

This concludes the discussion of the case $a_{i}=1$.

III. $a_{i}>1, a_{i} \leqslant s_{i}\left(2 a_{i} \leqslant b_{i+1}\right)$

In this case $b_{i+1} \geqslant 2$ and $M_{\Phi_{i}}^{(k)} \neq \phi$ for $k=1,2,3$.

1) $m \in M_{\Phi_{i}}^{(1)}, m=q_{i-1}+\left(s_{i}+k\right) q_{i}+\sum_{j=i+n} r_{j} q_{j}, 1 \leqslant k \leqslant a_{i}-1, n \geqslant 1$ :

For $1<k<a_{i}-1$ we get

$$
\begin{aligned}
& m_{L}=m-g_{i} \in M_{\Phi_{i}}^{(1)}: d_{L}=q_{i} \\
& m_{R}=m+q_{i} \in M_{\Phi_{i}}^{(1)}: d_{R}=q_{i} .
\end{aligned}
$$

For $k=1$ and therefore $m=q_{i-1}+\left(s_{i}+1\right) q_{i}+\sum_{j=i+n} r_{j} q_{j}, n \geqslant 1$

we find

$$
\begin{aligned}
& m_{L}=q_{i-1}+s_{i} q_{i}+\sum_{j=i+n} r_{j} q_{j} \in M_{\Phi_{i}}^{(2)} \text { if } n \text { is even: } d_{L}=q_{i} \\
& m_{L}=a_{i} q_{i}+\sum_{j=i+n} r_{j} q_{j} \in M_{\Phi_{i}}^{(3)} \text { if } n \text { is odd: } d_{L}=q_{i+1}-\left(2 a_{i}-1\right) q_{i}
\end{aligned}
$$

and

$m_{R}=m+q_{i} \in M_{\Phi_{1}}^{(1)}: d_{R}=q_{i}$

For $k=a_{i}-1$ and hence $m=q_{i-1}+\left(b_{i+1}-1\right) q_{i}+\sum_{j=i+n} r_{j} q_{j}$ we get 


$$
\begin{aligned}
& m_{L}=m-q_{i} \in M_{\Phi_{i}}^{(1)}: d_{L}=q_{i} \\
& m_{R}=\left(r_{i+1}+1\right) q_{i+1}+\sum_{j=i+2} r_{j} q_{j} \in M_{\Phi_{i}}^{(3)} \text { if } n=1: d_{R}=q_{i} \\
& m_{R}=q_{i+1}+\sum_{j=i+n} r_{j} q_{j} \in M_{\Phi_{i}}^{(3)} \text { if } n \geqslant 3 \text { or } n=2 \text { and } r_{i+2}<b_{i+3}: \\
& d_{R}=q_{i}, \\
& m_{R}=\left(r_{i+2 k-1}+1\right) q_{i+2 k-1}+\sum_{j=i+2 k} r_{j} q_{j} \in M_{\Phi_{i}}^{(3)} \text { if } r_{i+2}=b_{i+3} \text { and } \\
& k=\min \left\{l: r_{i+2 l}<b_{i+2 l-1}\right\}: d_{R}=q_{i} .
\end{aligned}
$$

2) $m \in M_{\Phi_{i}}^{(2)}$ with $m=q_{i-1}+s_{i} q_{i}+\sum_{j=i+2 n} r_{j} q_{j}, n \geqslant 1$. In this case we find:

$$
\begin{aligned}
& m_{L}=a_{i} q_{i}+\sum_{j=i+2 n} r_{j} q_{j} \in M_{\Phi_{i}}^{(3)}: d_{L}=q_{i+1}-2 a_{i} q_{i} \\
& m_{R}=m+q_{i} \in M_{\Phi_{i}}^{(2)}: d_{R}=q_{i} .
\end{aligned}
$$

3) $m \in M_{\Phi_{i}}^{(3)}, m=\sum_{j=i+n} r_{j} q_{j}, n \geqslant 0$ and $1 \leqslant r_{i} \leqslant a_{i}$ in the case $n=0$.

If $n=0$ and $1 \leqslant r_{i}<a_{i}$ one finds

$m_{L}=m-q_{i} \in M_{\Phi_{i}}^{(3)}: d_{L}=q_{i}$

$m_{R}=m+q_{i} \in M_{\Phi_{i}}^{(3)}: d_{R}=q_{i}$.

If $n=0$ and $r_{i}=a_{i}$ we get

$m_{L}=m-q_{i} \in M_{\Phi_{i}}^{(3)}: d_{L}=q_{i}$

$m_{R}=q_{i-1}+\left(s_{i}+1\right) q_{i}+\sum_{j=i+k} r_{j} q_{j} \in M_{\Phi_{i}}^{(1)}$ for $k$ odd: $d_{R}=q_{i+1}-\left(2 a_{i}-1\right) q_{i}$

respectively

$m_{R}=q_{i-1}+s_{i} q_{i}+\sum_{j=i+k} r_{j} q_{j} \in M_{\Phi_{1}}^{(2)}$ for $k$ even, $k \geqslant 2: d_{R}=q_{i+1}-2 a_{i} q_{i}$.

If $n=1$ and hence $m=r_{i+1} q_{i+1}+\sum_{j=i+2} r_{j} q_{j}$ one gets

$$
\begin{aligned}
& m_{L}=q_{i-1}+\left(b_{i+1}-1\right) q_{i}+m-q_{i+1} \in M_{\boldsymbol{\Phi}_{i}}^{(1)}: d_{L}=q_{i} \\
& m_{R}=m+q_{i} \in M_{\Phi_{i}}^{(3)} \text { if } r_{i+1} \neq b_{i+2}: d_{R}=q_{i} \\
& m_{R}=\left(r_{i+2}+1\right) q_{i+2}+\sum_{j=i+3} r_{j} q_{j} \in M_{\boldsymbol{\Phi}_{i}}^{(3)} \text { if } r_{i+1}=b_{i+2}: d_{R}=q_{i} .
\end{aligned}
$$


If $n \geqslant 2$ and hence $m=\sum_{j=i+n} r_{j} q_{j}$ we get for $n$ even $m_{L}=\sum_{j=1}^{n / 2} b_{i+2 j} q_{i+2 j-1}+\left(r_{i+n}-1\right) q_{i+n}+\sum_{j=i+n+1} r_{j} q_{j} \in M_{\Phi_{i}}^{(3)}: d_{L}=q$, whereas for $n$ odd one finds

$$
\begin{aligned}
m_{L} & =q_{i-1}+\left(b_{i+1}-1\right) q_{i}+\sum_{j=1}^{(n-1) / 2} b_{i+1+2 j} q_{i+2 j}+\left(r_{i+n}-1\right) q_{i+n} \\
& +\sum_{j=i+n+1} r_{j} q_{j} \in M_{\Phi_{i}}^{(1)}: d_{L}=q_{i}
\end{aligned}
$$

$m_{R}=m+q_{i} \in M_{\Phi_{i}}^{(3)}: d_{R}=q_{i}$ both for odd and even $n$.

IV. $a_{i}>1, a_{i} \geqslant s_{i}+1\left(2 a_{i} \geqslant b_{i+1}+1\right)$

1) $m \in M_{\Phi_{i}}^{(1)}: m=q_{i-1}+\left(s_{i}+k\right) q_{i}+\sum_{j=i+n} r_{j} q_{j}, 1 \leqslant k \leqslant a_{i}-1, n \geqslant 1$.

If $k=1 \neq a_{i}-1$ then

$m_{L}=\left(s_{i}+1\right) q_{i}+\sum_{j=i+n} r_{j} q_{j} \in M_{\Phi_{i}}^{(3)}: d_{L}=q_{i-1}$

$m_{R}=\left(s_{i}+2\right) q_{i}+\sum_{j=i+n} r_{j} q_{j} \in M_{\Phi_{i}}^{(3)}$ if $a_{i}>s_{i}+1: d_{R}=q_{i}-q_{i-1}$

respectively

$m_{R}=m+q_{i} \in M_{\Phi_{1}}^{(1)}$ if $a_{i}=s_{i}+1: d_{R}=q_{i}$.

If $k=1=a_{i}-1$ and hence $a_{i}=2$ we see from $a_{i} \geqslant s_{i}+1$ that $b_{i+1}=3$

and therefore $m=q_{i-1}+2 q_{i}+\sum_{j=i+n} r_{j} q_{j}, n \geqslant 1$. Hence we get

$$
\begin{aligned}
& m_{L}=2 q_{i}+\sum_{j=i+n} r_{j} q_{j} \in M_{\Phi_{i}}^{(3)}: d_{L}=q_{i-1} \\
& m_{R}=q_{i+1}+\sum_{j=i+n} r_{j} q_{j} \in M_{\Phi_{i}}^{(3)}: d_{R}=q_{i} .
\end{aligned}
$$

If $k \geqslant 2$ we get for $k=a_{i}-1: m=q_{i-1}+\left(b_{i+1}-1\right) q_{i}+\sum_{j=i+n} r_{j} q_{j}, n \geqslant 1$

and hence

$$
\begin{aligned}
& m_{L}=q_{i-1}+\left(b_{i+1}-2\right) q_{i}+\sum_{j=i+n} r_{j} q_{j} \in M_{\Phi_{i}}^{(1)}: d_{L}=q_{i} \\
& m_{R}=b_{i+1} q_{i}+\sum_{j=i+n} r_{j} q_{j} \in M_{\Phi_{i}}^{(3)}, \text { if } a_{i}=b_{i+1}: d_{R}-q_{i-1}
\end{aligned}
$$




$$
\begin{aligned}
& m_{R}=q_{i+1}+\sum_{j=i+n} r_{j} q_{j} \in M_{\Phi_{i}}^{(3)}, \text { if } a_{i}<b_{i+1} \text { and } n=1 \text { or } n=3: d_{R}=q_{i} \\
& m_{R}=q_{i+1}+\sum_{j=i+2} r_{j} q_{j} \in M_{\Phi_{t}}^{(3)}, \text { if } a_{i}<b_{i+1}, n=2 \text { and } r_{i+2} \leqslant b_{i+3}-1: \\
& d_{R}=q_{i} \\
& m_{R}=\left(r_{i+3}+1\right) q_{i+3}+\sum_{j=i+4} r_{j} q_{j} \in M_{\Phi_{i}}^{(3)} \text {, if } a_{i}<b_{i+1}, n=2, \\
& r_{i+2}=b_{i+3}: d_{R}=q_{i} .
\end{aligned}
$$

Remains for $m \in M_{\Phi_{1}}^{(1)}$ the case $1<k<a_{i}-1$ :

if $s_{i}+k \geqslant a_{i}+1$ then

$$
m_{L}=q_{i-1}+\left(s_{i}+k-1\right) q_{i}+\sum_{j=i+n} r_{j} q_{j} \in M_{\Phi_{i}}^{(1)}: d_{L}=q_{i}
$$

where as for $s_{i}+k \leqslant a_{i}$

$$
m_{L}=\left(s_{i}+k\right) q_{i}+\sum_{j=i+n} r_{j} q_{j} \in M_{\Phi_{i}}^{(3)}: d_{L}=q_{i-1} .
$$

The corresponding right neighbours are

$$
\begin{aligned}
& m_{R}=m+q_{i} \in M_{\Phi_{i}}^{(1)} \text { if } s_{i}+k \geqslant a_{i}: d_{R}=q_{i} \\
& m_{R}=\left(s_{i}+k+1\right) q_{i}+\sum_{j=i+n} r_{j} q_{j} \in M_{\boldsymbol{\Phi}_{i}}^{(3)} \text { if } s_{i}+k \leqslant a_{i}-1: d_{R}=q_{i}-q_{i-1} .
\end{aligned}
$$

2) $m \in M_{\Phi_{i}}^{(2)}: m=q_{i-1}+s_{i} q_{i}+\sum_{j=i+2 n} r_{j} q_{j}, n \geqslant 1$

For $a_{i} \geqslant s_{i}+1$ one finds

$$
\begin{aligned}
& m_{L}=s_{i} q_{i}+\sum_{j=i+2 n} r_{j} q_{j} \in M_{\Phi_{i}}^{(3)}: d_{L}=q_{i-1} \\
& m_{R}=\left(s_{i}+1\right) q_{i}+\sum_{j=i+2 n} r_{j} q_{j} \in M_{\Phi_{i}}^{(3)}: d_{R}=q_{i}-q_{i-1}
\end{aligned}
$$

3) $m \in M_{\boldsymbol{\Phi}_{i}}^{(3)}: m=\sum_{j=i+n} r_{j} q_{j}, n \geqslant 0$ and $1 \leqslant r_{i} \leqslant a_{i}$ if $n=0$.

If $n=0,1 \leqslant r_{i} \leqslant a_{i}$ we therefore have $m=r_{i} q_{i}+\sum_{j=i+l} r_{j} q_{j}, l \geqslant 1$.

For $l=1$ we find

$$
m_{L}=\left(r_{i}-1\right) q_{i}+\sum_{j=i+l} r_{j} q_{j} \in M_{\Phi_{i}}^{(3)} \text { if } r_{i} \leqslant s_{i}+1: d_{L}=q_{i}
$$


$m_{L}=q_{i-1}+\left(r_{i}-1\right) q_{i}+\sum_{j=i+l} r_{j} q_{j} \in M_{\Phi_{l}}^{(1)}$ if $r_{i}>s_{i}+1: d_{L}=q_{i}-q_{i-1}$

For $l \geqslant 2$ we find

$m_{L}=\left(r_{i}-1\right) q_{i}+\sum_{j=i+l} r_{j} q_{j} \in M_{\boldsymbol{\Phi}_{i}}^{(3)}, 1 \leqslant r_{i} \leqslant s_{i}: d_{L}=q_{i}$

$m_{L}=q_{i-1}+s_{i} q_{i}+\sum_{j=i+l} r_{j} q_{j} \in M_{\Phi_{i}}^{(2)}, r_{i}=s_{i}+1, l$ even: $d_{L}=q_{i}$

$m_{L}=s_{i} q_{i}+\sum_{j=i+l} r_{j} q_{j} \in M_{\Phi_{i}}^{(3)}, r_{i}=s_{i}+1, l$ odd: $d_{L}=q_{i}-q_{i-1}$

$m_{L}=q_{i-1}+\left(r_{i}-1\right) q_{i}+\sum_{j=i+l} r_{j} q_{j} \in M_{\Phi_{i}}^{(1)}: r_{i} \geqslant s_{i}+2: d_{L}=q_{i}-q_{q-1}$.

For the right neighbour we find in the case $n=0$ and $l=1$ :

$m_{R}=\left(r_{i}+1\right) q_{i}+\sum_{j=i+1} r_{j} q_{j} \in M_{\Phi_{i}}^{(3)}$ if $r_{i}<s_{i}+1: d_{R}=q_{i}$

$m_{R}=q_{i-1}+m \in M_{\Phi_{i}}^{(1)}$, if $r_{i}=s_{i}+1: d_{R}=q_{i-1}$

$m_{R}=q_{i-1}+m \in M_{\Phi_{i}}^{(1)}$ if $r_{i}>s_{i}+1, r_{i} \leqslant a_{i}<b_{i+1}$ or $r_{i}<a_{i}=b_{i+1}$ :

$d_{R}=q_{i-1}$

$m_{R}=\left(r_{i+1}+1\right) q_{i+1}+\sum_{j=i+2} r_{j} q_{j} \in M_{\Phi_{i}}^{(3)}$ if $r_{i}=a_{i}=b_{i+1}: d_{R}=q_{i-1}$.

For $l \geqslant 2$ we find for $1 \leqslant r_{i} \leqslant s_{i}-1$

$m_{R}=\left(r_{i}+1\right) q_{i}+\sum_{j=i+l} r_{j} q_{j} \in M_{\Phi_{i}}^{(3)}: d_{R}=q_{i}$

$m_{R}=q_{i-1}+s_{i} q_{i}+\sum_{j=i+l} r_{j} q_{j} \in M_{\Phi_{i}}^{(2)}$ if $r_{i}=s_{i}, l$ even: $d_{R}=q_{i-1}$

$m_{R}=m+q_{i} \in M_{\Phi_{1}}^{(3)}$ if $r_{i}=s_{i}, l$ odd: $d_{R}=q_{i}$.

For $r_{i}=s_{i}+1$ we find

$m_{R}=q_{i-1}+\left(s_{i}+1\right) q_{i}+\sum_{j=i+l} r_{j} q_{j} \in M_{\Phi_{i}}^{(1)}: d_{R}=q_{i-1}$.

If $l \geqslant 2$ and $a_{i} \geqslant r_{i}>s_{i}+1$ we see that

$m_{R}=q_{i-1}+m \in M_{\Phi_{i}}^{(1)}$ if $r_{i} \neq b_{i+1}: d_{R}=q_{i-1}$

$m_{R}=q_{i+1}+\sum_{j=i+l} r_{j} q_{j} \in M_{\Phi_{i}}^{(3)}$ if $r_{i}=a_{i}=b_{i+1}, r_{i+2}<b_{i+3}$ : 
$d_{R}=q_{i-1}$

$m_{R}=\left(r_{i+2 k-1}+1\right) q_{i+2 k-1}+\sum_{j=i+2 k} r_{j} q_{j} \in M_{\Phi_{i}}^{(3)}$ if $r_{i}=a_{i}=b_{i+1}$

and $k=\min \left\{l: r_{i+2 l}<b_{i+2 l+1}\right\}: d_{R}=q_{i-1}$.

If $n=1$ we have $m=\sum_{j=i+1} r_{j} q_{j}$ and hence

$m_{L}=q_{i-1}+\left(b_{i+1}-1\right) q_{i}+\left(r_{i+1}-1\right) q_{i+1}$

$+\sum_{j=i+2} r_{j} q_{j} \in M_{\Phi_{i}}^{(1)}: d_{L}=q_{i}$

$m_{R}=q_{i}+m \in M_{\Phi_{i}}^{(3)}$ if $r_{i+1} \leqslant b_{i+2}-1: d_{R}=q_{i}$

$m_{R}=\left(r_{i+2 k-1}+1\right) q_{i+2 k-1}+\sum_{j=i+2 k} r_{j} q_{j} \in M_{\Phi_{i}}^{(3)}$, if $r_{i+1}=b_{i+2}$ and

$k=\min \left\{l: r_{i+2 l-1}<b_{i+2 l}\right\}: d_{R}=q_{i}$.

If $n \geqslant 2$ we have for even $n$ :

$m_{L}=\sum_{j=1}^{n / 2} b_{i+2 j} q_{i+2 j-1}+\left(r_{i+n}-1\right) q_{i+n}+\sum_{j=i+n+1} r_{j} q_{j} \in M_{\Phi_{1}}^{(3)}$

$d_{L}=q_{i}$

and for $n$ odd

$$
\begin{aligned}
m_{L} & =q_{i-1}+\left(b_{i+1}-1\right) q_{i}+\sum_{j=1}^{(n-1) / 2} b_{i+1+2 j} q_{i+2 j}+\left(r_{i+n}-1\right) q_{i+n} \\
& +\sum_{j=i+n+1} r_{j} q_{j} \in M_{\Phi_{i}}^{(1)}: d_{L}=q_{i} \\
m_{R} & =q_{i}+m \in M_{\Phi_{i}}^{(3)}: d_{R}=q_{i} .
\end{aligned}
$$

This then proves Prop.7. The proofs of Prop. 8 respectively 9 proceed along the same lines.

\section{References}

[1] Lohöfer, G., and Mayer, D., Correlation functions of a time continuous dissipative system with a strange attractor, Phys. Lett., 113A (1985), 105-110.

[2] Florek, K., Une remarque sur la repartition des nombres $n \xi$ mod 1, Coll. Math. Wroclaw, 2 (1951), 323-324.

[ 3 ] Slater, N. B., Gaps and steps for the sequence $n \theta \bmod 1$, Proc. Camb. Phil. Soc., 63 (1967), $1115-1123$. 
[ 4 ] Birkhoff, G., Proof of a recurrence theorem for strongly transitive systems, Proc. Nat. Acad. Sci. USA, 17 (1931), 650-655.

[ 5 ] Kac. M., Probability and Related Topics in Physical Sciences, Interscience Publishers LTD, London, 1959.

Smoluchowski, R., Drei Vorträge über Diffusion, Brown-sche Molekularbewegung und Koagulation von Kolloidteilchen, Phys. Z., 17 (1916), 557-571, 587-599.

[6] Slater, N. B., The distribution of the integers $N$ for which $\{\theta N\} \subset \Phi$, Proc. Camb. Phil. Soc., 46 (1950), 525-534.

[ 7 ] Lohöfer, G., Korrelationsfunktionen eines zeitlich kontinuierlichen, chaotischen Systems mit seltsamem Attraktor, Ph.-D Thesis RWTH Aachen (1986), unpublished.

[ 8 ] Coquet, J., Rhin, G., and Toffin, Ph., Représentation des entiers naturels et indépendence statistique, Ann. Inst. Fourier, Grenoble, 31 (1981), 1-15.

[ 9 ] Lang, S., Introduction to Diophantine approximations, Addison Wesley, London 1966.

[10] Khinchin, A. Ya., Continued fractions, Univ. of Chicago Press, Chicago, 1964.

[11] Dupain, Y., and Sos, V., On the one-sided boundedness of discrepency function of the sequence $\{n \alpha\}$, Acta Arithmetica, XXXVII (1980), 363-374. 
